\title{
Proteomic analysis of protein expression profiles during hyperthermia-induced apoptosis in Tca8113 cells
}

\author{
WEN JIANG ${ }^{1,2^{*}}$, LI BIAN $^{3 *}$, NING WANG ${ }^{1}$ and YONGWEN HE ${ }^{1}$ \\ ${ }^{1}$ Department of Dental Research, The Affiliated Stomatological Hospital of Kunming Medical University, Kunming, \\ Yunnan 650031; ${ }^{2}$ The First Affiliated Hospital of Yangtze University, Jingzhou, Hubei 434000; \\ ${ }^{3}$ Department of Pathology, The First Affiliated Hospital of Kunming Medical University, \\ Kunming, Yunnan 650032, P.R. China
}

Received December 16, 2012; Accepted April 18, 2013

DOI: $10.3892 / \mathrm{ol} .2013 .1354$

\begin{abstract}
The aim of the present study was to explore protein expression profiles during cancer cell apoptosis induced by hyperthermia. A hyperthermia-induced apoptosis model was established using a Tca8113 cell line derived from a human tongue squamous cell carcinoma, which underwent fluorescent differential display two-dimensional (2D) gel electrophoresis at 2, 6, 8, 12 and $24 \mathrm{~h}$ following the induction of hyperthermia. Proteins were identified by mass spectrometry analysis. Expression changes in the proteins were detected by western blot analysis. A total of 107 proteins were detected that exhibited different expression levels in the hyperthermia-treated cells compared with the controls, and 57 of these proteins were identified. Expression changes in the representative proteins were further verified by western blot analysis. These 57 proteins were identified according to the following functional groups: energy metabolism-related enzymes, cytoskeleton-related proteins, chaperones, transcription factors, protein synthesis-related proteins and cell division- and proliferation-related proteins. These groups included 44 upregulated and 13 downregulated proteins. Among the 44 upregulated proteins, 27 were upregulated continuously, eight were upregulated at an early time-point and nine were upregulated at a middle to late time-point. Among the 13 downregulated proteins, five were downregulated continuously, six were downregulated at an early time-point and two were downregulated at a middle to late time-point. These results indicate that hyperthermia-induced Tca8113 cell
\end{abstract}

Correspondence to: Professor Yongwen He, Department of Dental Research, The Affiliated Stomatological Hospital of Kunming Medical University, 1168 West Chunrong Road, Yuhua Avenue, Chenggong, Kunming, Yunnan 650031, P.R. China

E-mail: heyongwen2@sina.com

*Contributed equally

Key words: proteomic, hyperthermia, apoptosis, cancer, mass spectrometry analysis apoptosis is controlled by multiple factors, which include time and regulatory proteins.

\section{Introduction}

Malignant tumors are treated with a combination of therapeutic modalities, including surgery, radiotherapy, chemotherapy, immunotherapy and hyperthermia therapy. Hyperthermia is effective in killing tumor cells; moreover, it is capable of increasing the sensitivity of tumor cells towards radiotherapy and numerous antitumor drugs (1). Therefore, hyperthermia is considered to be an effective and promising tumor therapy (2). Hyperthermia inhibits tumor cells through the five following mechanisms: i) by inhibiting tumor cell respiration, thereby increasing anaerobic glycolysis and increasing the acidity of the environment, which may result in damage to the tumor cell membranes and the release of lysosomal acid hydrolase; ii) by altering the enzyme structure of the cytoplasm and the nucleus, thereby leading to a metabolic disorder; iii) by inhibiting the synthesis of DNA, RNA and the associated proteins, thereby leading to the aggregation of denatured nuclear matrix proteins; iv) by impairing the cytoskeleton; and v) by inhibiting anti-apoptotic proteases and/or the activity of oncogenes, and enhancing pro-apoptotic proteases and/or tumor suppressor gene activity, thereby leading to tumor cell necrosis and/or the induction of apoptosis (3-6).

Hyperthermic temperatures between 40 and $45^{\circ} \mathrm{C}$ kill tumor cells, mainly by inducing apoptosis $(7,8)$. The therapeutic temperature for treating an oral tumor is $43^{\circ} \mathrm{C}$, which is used to treat malignant oral tumors by inducing tumor cell apoptosis. Hyperthermia-induced tumor cell apoptosis relies on numerous approaches and regulation factors. It has been demonstrated that hyperthermia is able to activate the release of pro-apoptotic factors, such as cytochrome $C$, apoptosis-inducing factor (AIF) and Smac/Diablo, from the mitochondria, and activate downstream effectors to induce apoptosis (9). In addition, hyperthermia has been demonstrated to enhance Fas-L, tumor necrosis factor- $\alpha$ (TNF- $\alpha$ ) and TNF-related apoptosis-inducing ligand (TRAIL) expression, and to increase tumor cell sensitivity towards their receptors, which ultimately induces apoptosis through the death receptor pathway (10-12). Furthermore, it has been indicated 
that hyperthermia induces intracellular reactive oxygen species (ROS) production and the activation of downstream effectors, which leads to apoptosis $(13,14)$. The treatment also promotes the influx of extracellular $\mathrm{Ca}^{2+}$ and/or the damage of the cytoplasmic calcium pool, thereby increasing intracellular $\mathrm{Ca}^{2+}$ concentrations and activating $\mathrm{Ca}^{2+}$-related enzymes, thus resulting in apoptosis $(15,16)$. Moreover, it has been demonstrated that hyperthermia is able to activate or upregulate the gene or protein expression of p53, Bax, Bak and caspase family members, again leading to apoptosis $(9,17-20)$. During hyperthermia-induced tumor cell apoptosis, anti-apoptotic protective factors are produced by the tumor cells for the maintenance of self-survival and for protecting the cell from damage. For example, heat shock proteins (HSPs) are produced under stress to maintain cell survival (21). It has been demonstrated that the expression of HSPs is increased significantly following hyperthermia (22-25), which may inhibit apoptosis. Additionally, it has been demonstrated that Bcl-2 and Mcl-1 are also able to protect tumor cells from hyperthermic damage $(26,27)$. However, the mechanism of hyperthermia-induced tumor cell apoptosis has not been fully elucidated.

In our previous study, we demonstrated that hyperthermia-induced Tca8113 cell apoptosis involved changes in the expression and function of multiple proteins (28). In the present study, a hyperthermia-induced apoptosis model was established using the Tca8113 cell line derived from a human tongue squamous cell carcinoma. Cell lysates were subjected to fluorescent differential display two-dimensional (2D) gel electrophoresis $2,6,8,12$ and $24 \mathrm{~h}$ after hyperthermia treatment. A total of 107 proteins were detected that exhibited different expression levels in the hyperthermia-treated cells compared with in the controls, using matrix-associated laser desorption/ionization (MALDI)-time of flight (TOF) or MALDI-TOF/TOF mass spectrometry analysis. This method allowed us to obtain the peptide mass fingerprint, and 57 proteins were identified by searching the SwissProt and NCBInr databases. Following the analysis of the protein profiles, the results indicated that hyperthermia-induced Tca8113 cell apoptosis is controlled by multiple factors, including time and regulatory proteins.

\section{Materials and methods}

Materials. The human tongue squamous cell carcinoma cell line, Tca8113, was purchased from Shanghai Cell Bank (Chinese Academy of Sciences, Shanghai, China). The CyDye Difference Gel Electrophoresis (DIGE) fluorescent Cy2, Cy3 and Cy5 were purchased from GE Healthcare (Uppsala, Sweden). The heat shock $70 \mathrm{kDa}$ protein (HSP70; D69), stathmin 1 and Lamin A/C polyclonal antibodies were purchased from Cell Signaling Technology, Inc. (Danvers, MA, USA). Cofilin 1 (5) sc-53934 mouse monoclonal $\mathrm{IgG}_{2 b}$ was purchased from Santa Cruz Biotechnology, Inc. (Santa Cruz, CA, USA). The anti-phosphoglycerate mutase 1 (PGAM1) antibody (rabbit polyclonal to PGAM1; ab96622) and the anti- $\Delta(3,5)-\Delta(2,4)$-dienoyl-CoA isomerase mitochondrial (ECH1p) antibody (rabbit polyclonal to ECH1p; ab90645) were purchased from Abcam (Cambridge, UK).

Cell culture and hyperthermia treatment. The Tca8113 cells were cultured in RPMI-1640 medium containing 10\% fetal bovine serum (with $1 \times 10^{5} \mathrm{U} / 1$ penicillin and $1 \times 10^{2} \mathrm{mg} / \mathrm{l}$ streptomycin) at $37^{\circ} \mathrm{C}$ with $5 \% \mathrm{CO}_{2}$. Cells at $80-90 \%$ confluence were treated in a temperature-controlled water bath for $40 \mathrm{~min}$ at $43^{\circ} \mathrm{C}$, and then cultured under normal conditions for $2,6,8$, 12 and $24 \mathrm{~h}$. The cells solely cultured under normal conditions were used as the control.

Sample preparation and quantitation. The Tca8113 cells $\left(2 \times 10^{7}\right)$ were collected and centrifuged at $228 \times \mathrm{g}$ for $5 \mathrm{~min}$. The subsequent cell pellets were washed with phosphate-buffered saline (PBS) and then centrifuged at $228 \mathrm{x}$ g for $5 \mathrm{~min}$. This was repeated three times. The cell pellets were resuspended in $250 \mu \mathrm{l}$ DIGE lysis buffer containing $7 \mathrm{M}$ urea, $2 \mathrm{M}$ thiourea, $65 \mathrm{mM}$ Tris, 4\% CHAPS, $0.2 \%$ IPG buffer and a protease inhibitor cocktail (Roche Diagnostics GmbH, Mannheim, Germany), which was sonicated briefly on ice. The cell lysates were centrifuged at $15,600 \mathrm{x}$ g for $20 \mathrm{~min}$ at $4^{\circ} \mathrm{C}$. The supernatant was collected as the cell lysate, and the protein concentration was measured with the Bio-Rad Protein Assay (Bio-Rad Laboratories, Hercules, CA, USA) and adjusted to the same concentration for each sample.

$2 D-D I G E$ assay. Electrophoresis was conducted as described previously (29), and was performed with a model E-600 electrophoresis system (Amersham, Piscataway, NJ, USA). The $\mathrm{pH}$ of the protein samples was set at 8.0-9.0, and the samples were diluted to $5 \mu \mathrm{g} / \mu \mathrm{l}$. All samples were mixed to equal amounts and aliquoted at $50 \mu \mathrm{g} / 10 \mu \mathrm{l}$, which was then used as the internal standard. A total of $50 \mu \mathrm{g}$ sample was labeled with $400 \mathrm{pmol} / \mu \mathrm{l}$ Cy3 and Cy5, respectively. The internal standard was labeled with $400 \mathrm{pmol} / \mu \mathrm{l} \mathrm{Cy} 2$. The three types of labeled samples were mixed evenly and the proteins were separated by isoelectric focusing electrophoresis (IFE), with a $\mathrm{pH}$ gradient of 3.0-10.0 on a 13-cm non-linear strip. The IFE conditions were as follows: $30 \mathrm{~V}$ for $12 \mathrm{~h}, 500 \mathrm{~V}$ for $1 \mathrm{~h}, 1,000 \mathrm{~V}$ for $1 \mathrm{~h}, 8,000 \mathrm{~V}$ for $8 \mathrm{~h}$ and $500 \mathrm{~V}$ for $4 \mathrm{~h}$. Following isoelectric focusing, IPG strips were placed in equilibrium buffer and the second electrophoresis stage [12.5\% sodium dodecyl sulfate-polyacrylamide gel electrophoresis (SDS-PAGE)] was subsequently conducted. The SDS-PAGE conditions were $15 \mathrm{~mA}$ for $20 \mathrm{~min}$ and $30 \mathrm{~mA}$ until the bromophenol blue front reached the bottom of the gel.

Gel scanning and software analysis. The gel pattern was scanned with a Typhoon scanner (GE Healthcare Biosciences, Pittsburgh,PA, USA) at wavelengths of 488,532 and $633 \mathrm{~nm}$ for Cy2, Cy3 and Cy5, respectively, and optimized by ImageQuant software (GE Healthcare Biosciences). Differential points were analyzed by DeCyder ${ }^{\mathrm{TM}}$ 2D software, version $6.5(\mathrm{GE}$ Healthcare).

Protein identification. The gels were stained with Coomassie brilliant blue R350 (Amersham). The differential points were removed and the stain was removed in $\mathrm{NH}_{4} \mathrm{HCO}_{3} / 30 \% \mathrm{ACN}$ buffer, prior to being digested in trypsin for $20 \mathrm{~h}$. Peptides were extracted, desalted with a ZipTip (Millipore Corporation, Billerica, MA, USA) and analyzed by a 4800 Plus MALDI TOF/TOF ${ }^{\mathrm{TM}}$ analyzer (Applied Biosystems, Foster City, CA, USA). The differentially expressed proteins were identified by software analysis, as well as SwissProt and NCBInr database searches. 
Table I. Upregulation of 44 proteins during the $24 \mathrm{~h}$ following hyperthermia.

\begin{tabular}{|c|c|c|}
\hline Upregulated continuously & Upregulated early & Upregulated at a middle to late period \\
\hline$\alpha$-enolase & BolA-like protein 2 & Peroxiredoxin-6 \\
\hline Actin, cytoplasmic 2 & Cofilin-1 & Phosphoglycerate mutase 1 \\
\hline $\begin{array}{l}\text { Crystal structure of Ufc1, } \\
\text { the Ufm1-conjugating enzyme } 1 \text {, } \\
\text { chain A }\end{array}$ & $\begin{array}{l}\text { Deoxyuridine } 5 \text { '-triphosphate } \\
\text { nucleotidohydrolase, mitochondrial }\end{array}$ & $\begin{array}{l}\text { Human eukaryotic translation } \\
\text { initiation factor } 1 \mathrm{~A} \text {, chain } \mathrm{A}\end{array}$ \\
\hline F-actin-capping protein subunit $\beta$ & Glutathione S-transferase P & T-complex protein 1 subunit $\zeta$ \\
\hline $\begin{array}{l}26 \mathrm{~S} \text { proteasome non-ATPase } \\
\text { regulatory subunit } 14\end{array}$ & $\begin{array}{l}\text { Far upstream element-binding } \\
\text { protein } 1\end{array}$ & Splicing factor U2AF $65 \mathrm{kDa}$ subunit \\
\hline $\begin{array}{l}\text { Glyceraldehyde-3-phosphate } \\
\text { dehydrogenase }\end{array}$ & Peroxiredoxin-2 & $\begin{array}{l}\text { Scavenger mRNA-decapping } \\
\text { enzyme DcpS }\end{array}$ \\
\hline Heat shock protein $\beta-1$ & Pyruvate kinase isozymes M1/M2 & Keratin, type II cytoskeletal 8 \\
\hline Inorganic pyrophosphatase & Tubulin $\beta$ chain & $\begin{array}{l}60 \mathrm{kDa} \text { heat shock protein, } \\
\text { mitochondrial }\end{array}$ \\
\hline $\begin{array}{l}\text { Heterogeneous nuclear } \\
\text { ribonucleoprotein } \mathrm{H}\end{array}$ & & $\begin{array}{l}\text { 3-hydroxyacyl-CoA dehydrogenase } \\
\text { type-2 isoform } 1\end{array}$ \\
\hline \multicolumn{3}{|l|}{ Mitotic checkpoint protein BUB3 } \\
\hline \multicolumn{3}{|l|}{ Phosphoglycerate kinase 1} \\
\hline \multicolumn{3}{|l|}{ hCG15971, isoform CRA_b } \\
\hline \multicolumn{3}{|l|}{ Protein disulfide-isomerase A3 } \\
\hline \multicolumn{3}{|l|}{ Proteasome activator complex subunit 1} \\
\hline \multicolumn{3}{|l|}{ Peroxiredoxin-4 } \\
\hline \multicolumn{3}{|l|}{ Serum albumin } \\
\hline \multicolumn{3}{|l|}{ Flavin reductase } \\
\hline \multicolumn{3}{|l|}{ Galactokinase } \\
\hline \multicolumn{3}{|l|}{ Prohibitin } \\
\hline \multicolumn{3}{|l|}{ Protein 4.1 , isoform 3} \\
\hline \multicolumn{3}{|l|}{ Pyridoxine-5'-phosphate oxidase } \\
\hline \multicolumn{3}{|l|}{ ATP synthase subunit $d$, mitochondrial } \\
\hline \multicolumn{3}{|l|}{ Heat shock cognate $71 \mathrm{kDa}$ protein } \\
\hline \multicolumn{3}{|l|}{ Heat shock $70 \mathrm{kDa}$ protein 1} \\
\hline \multicolumn{3}{|l|}{ Keratin, type I cytoskeletal 10} \\
\hline \multicolumn{3}{|l|}{ Keratin, type II cytoskeletal 7} \\
\hline Keratin, type II cytoskeletal 1 & & \\
\hline
\end{tabular}

Western blot analysis. Equal amounts of protein were separated by $10 \%$ SDS-PAGE and electroblotted onto Immobilon-P membranes (Millipore Corporation). The membranes were incubated with the primary antibody at $4{ }^{\circ} \mathrm{C}$ overnight and then incubated with horseradish peroxidase-conjugated secondary antibody at room temperature for $1 \mathrm{~h}$. Following washing with $1 \mathrm{X}$ Tris-buffered saline with $0.1 \%$ Tween-20 (TBST), the membranes were visualized by chemiluminescence (Pierce, Biotechnology, Inc., Rockford, IL, USA). The relative protein content is the ratio of the test protein gray scale value in contrast with the $\alpha$-tubulin gray scale value, measured by ImageJ software (version 2.1.4.6; National Institutes of Health, Bethesda, MD, USA).

Statistical analysis. Differences in the protein expression profile were analyzed by DeCyder 2D software, version 6.5, and a Student's t-test was applied. Differences among the 6 groups were compared using a one-way ANOVA. $\mathrm{P}<0.01$ was considered to indicate a statistically significant difference.

\section{Results}

2D-DIGE gel pattern and analysis of differential protein points. The Tca8113 cells were incubated for $40 \mathrm{~min}$ in a water bath at $43^{\circ} \mathrm{C}$, and subsequently transferred to normal culture conditions for 2, 6, 8, 12 and $24 \mathrm{~h}$. Protein samples were labeled with $\mathrm{Cy} 2, \mathrm{Cy} 3$ and $\mathrm{Cy} 5$, which were separated by 2D-DIGE, and the respective blue, green and red gel images were captured for each dye (Fig. 1). The gel images were analyzed by DeCyder 2D software, version 6.5, and the data were compared by a one-way statistical analysis, which identified 107 differentially expressed proteins ( $\mathrm{P}=0.000$; Fig. 2). 
Table II. Downregulation of 13 proteins during the $24 \mathrm{~h}$ following hyperthermia.

Downregulated continuously

ATP synthase subunit $\alpha$, mitochondrial

Crystal structure of the human

eukaryotic translation initiation factor

$5 \mathrm{~A}$, chain A

Fructose-bisphosphate aldolase A

Vimentin

Stathmin 1/oncoprotein 18
Downregulated early

Downregulated at a middle to late period

Argininosuccinate synthase, mitochondrial

D-3-phosphoglycerate dehydrogenase Lamin-A/C

$\Delta(3,5)-\Delta(2,4)$-dienoyl-coenzyme A isomerase, mitochondrial

Eukaryotic translation initiation factor $5 \mathrm{~A}-1$

Proliferation-associated protein 2G4

Mitochondrial succinyl-CoA:3-

ketoacid-coenzyme A transferase 1
A

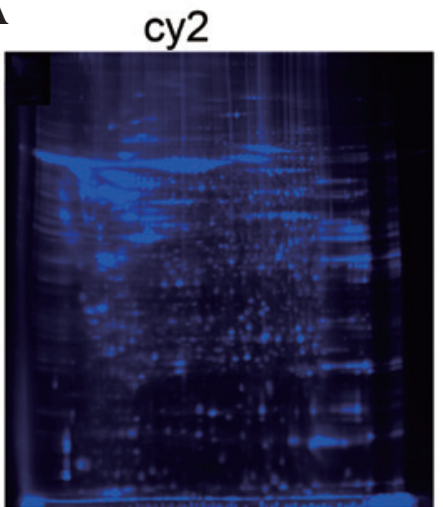

B

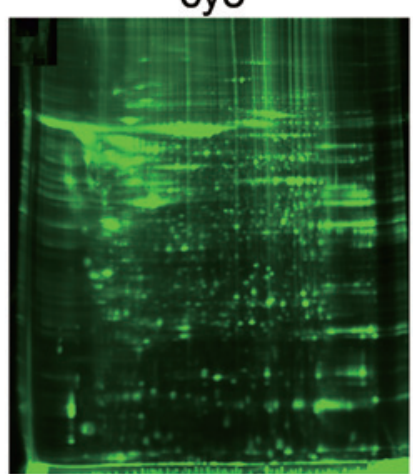

C

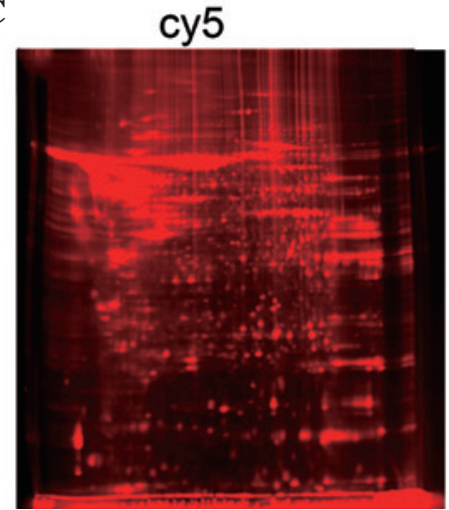

D

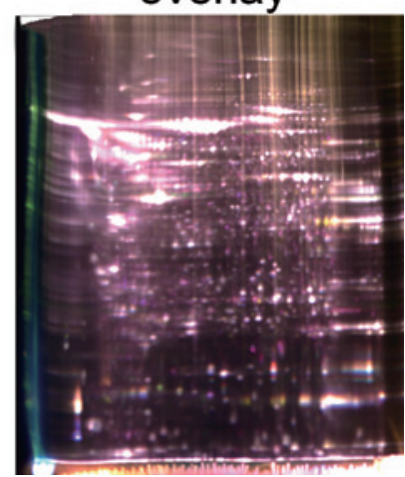

Figure 1. Protein fluorescence and overlay images. Protein samples were labeled with Cy2, Cy3 and Cy5, which were separated by two-dimensional differential in gel electrophoresis (DIGE), and the respective blue, green and red gel images were captured for each dye. (A) The image of the protein sample (labeled with Cy2) of the Tca8113 cells cultured under normal conditions. (B) The image of the protein sample (labeled with Cy3) of the Tca8113 cells treated for 40 min at $43^{\circ} \mathrm{C}$ and then cultured under normal conditions for $2 \mathrm{~h}$. (C) The image of the protein sample (labeled with Cy5) of the Tca8113 cells treated for 40 min at $43^{\circ} \mathrm{C}$ and then cultured under normal conditions for $6 \mathrm{~h}$. (D) Overlay of images A, B and C.
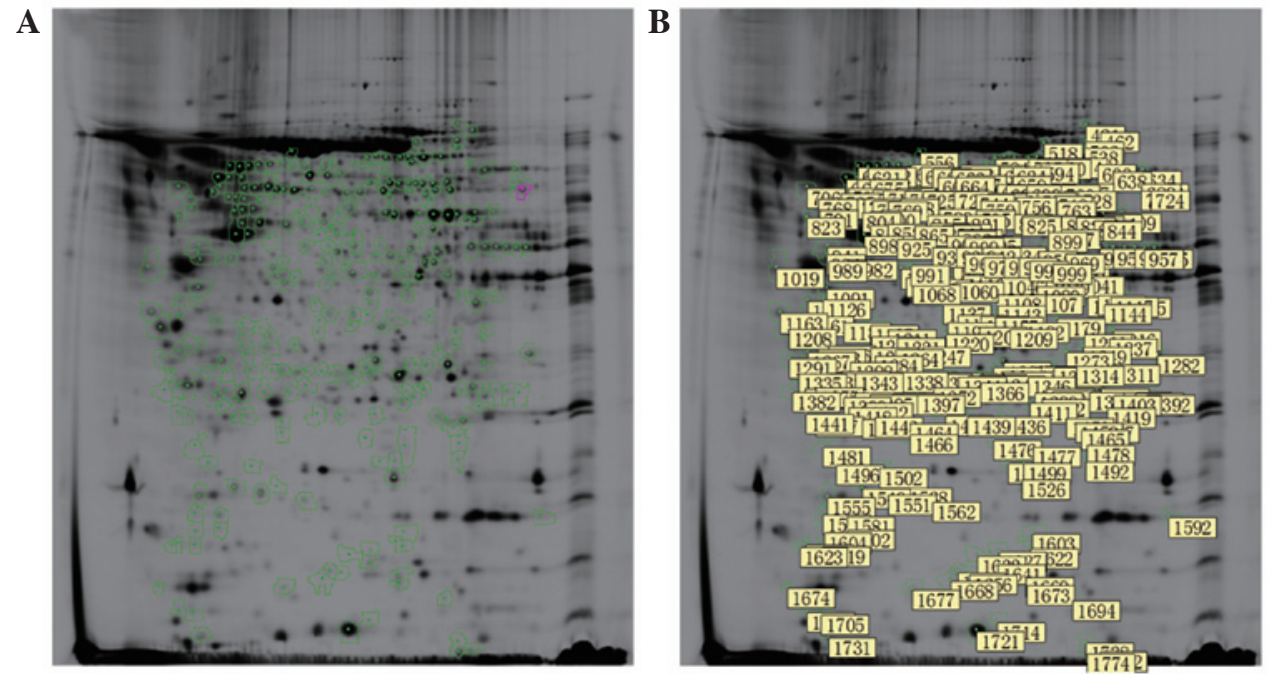

Figure 2. Differentially expressed protein points. The gel images were analyzed by DeCyder $2 \mathrm{D}$ software, version 6.5 , and the data were compared by a one-way statistical analysis, which identified 107 differentially expressed proteins. (A) The 107 differentially expressed protein points. (B) The master numbers of the 107 differentially expressed protein points. 
A

a. Master NO: 904

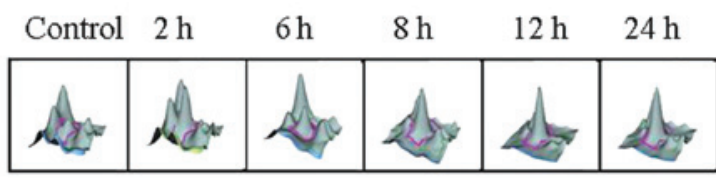

b. Master NO: 1526

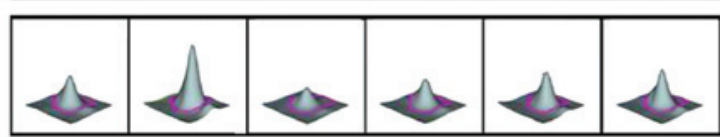

c. Master NO:1310

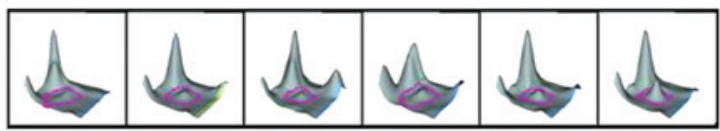

d. Master NO:1551

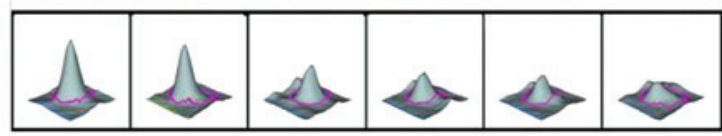

e. Master NO: 1209

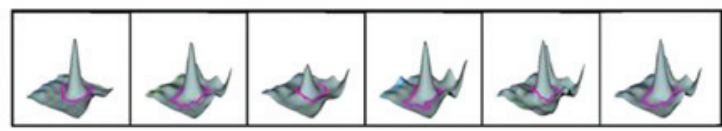

f. Master NO:584

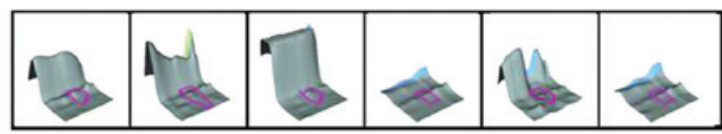

B
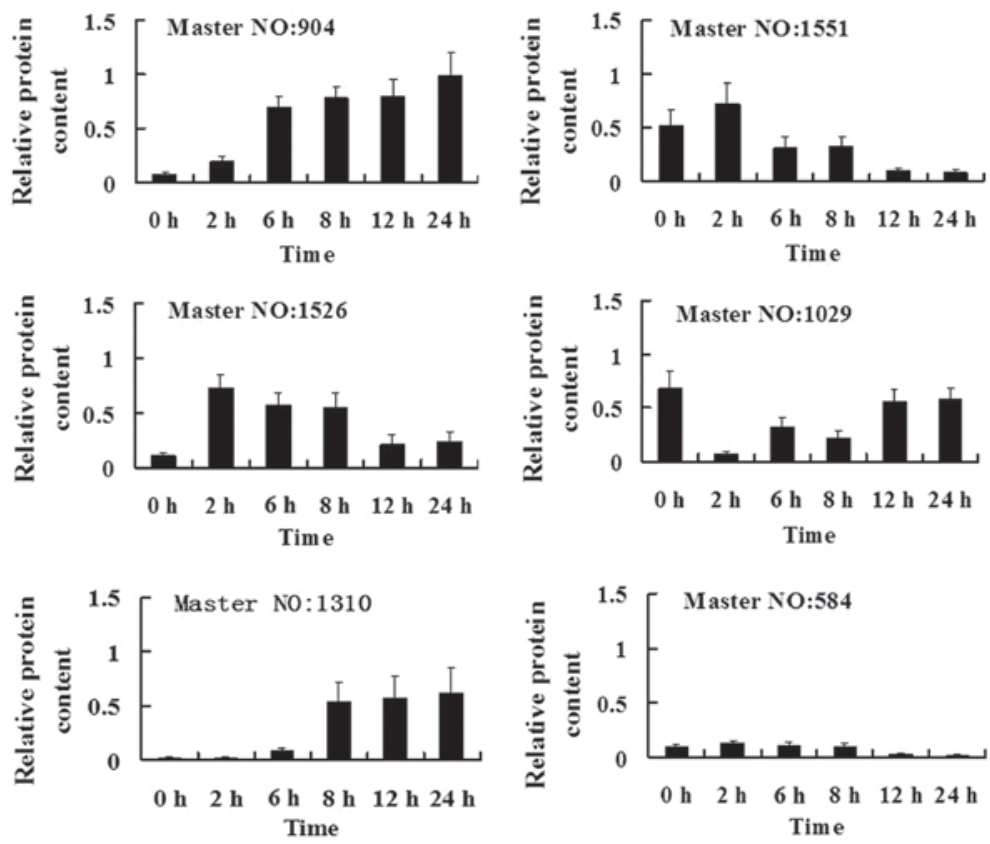

Figure 3. Dynamic changes in the expression levels of the differentially expressed proteins. (Aa). Master no. 904; heat shock $70 \mathrm{kDa}$ protein 1 was upregulated continuously. (Ab) Master no. 1526; cofilin-1 was upregulated during the early time period. (Ac) Master no. 1310; phosphoglycerate mutase 1 was upregulated during the middle to late time period. (Ad) Master no. 1551; stathmin 1/oncoprotein 18 was downregulated continuously. (Ae) Master no. 1209; $\Delta(3,5)-\Delta(2,4)$-dienoyl-CoA isomerase, mitochondrial was downregulated during the early time period. (Af) Master no. 584; lamin-A/C was downregulated during the middle to late time period. (B) The relative content of representative proteins to $\alpha$-tubulin following heating for $0,2,6,8,12$ and $24 \mathrm{~h}$, respectively. Data represent the mean from three independent experiments (bars, mean \pm standard deviation).

Differential protein point identification and dynamic expression analysis. A total of 107 differential protein points were detected by tandem mass spectroscopy and analyzed using the SwissProt and NCBInr databases, which identified 57 types of proteins (Tables I and II). The early period was considered to be within $8 \mathrm{~h}$ of hyperthermic treatment, while $8-24 \mathrm{~h}$ post-treatment was considered to be the middle to late period. Protein expres- sion levels that were higher in the hyperthermia-treated cells compared with the controls were considered to be upregulated, while proteins with lower expression levels were considered to be downregulated. The dynamic expression changes in 57 proteins, at 2, 6, 8, 12 and $24 \mathrm{~h}$ after hyperthermic treatment, were characterized. In total, 44 proteins were demonstrated to be upregulated and 13 proteins were shown to be downregulated. 
Table III. The relative content of representative proteins following heating for different time periods.

\begin{tabular}{|c|c|c|c|c|c|c|}
\hline Master no. & $0 \mathrm{~h}$ & $2 \mathrm{~h}$ & $6 \mathrm{~h}$ & $8 \mathrm{~h}$ & $12 \mathrm{~h}$ & $24 \mathrm{~h}$ \\
\hline 904 & $0.07 \pm 0.02$ & $0.19 \pm 0.05$ & $0.69 \pm 0.11$ & $0.78 \pm 0.11$ & $0.80 \pm 0.15$ & $0.99 \pm 0.20$ \\
\hline 1526 & $0.11 \pm 0.03$ & $0.73 \pm 0.12$ & $0.57 \pm 0.11$ & $0.55 \pm 0.13$ & $0.21 \pm 0.09$ & $0.24 \pm 0.08$ \\
\hline 1310 & $0.02 \pm 0.01$ & $0.02 \pm 0.01$ & $0.09 \pm 0.02$ & $0.54 \pm 0.17$ & $0.57 \pm 0.20$ & $0.62 \pm 0.23$ \\
\hline 1551 & $0.52 \pm 0.14$ & $0.72 \pm 0.19$ & $0.31 \pm 0.10$ & $0.33 \pm 0.09$ & $0.10 \pm 0.02$ & $0.08 \pm 0.03$ \\
\hline 1029 & $0.68 \pm 0.17$ & $0.07 \pm 0.02$ & $0.32 \pm 0.09$ & $0.22 \pm 0.07$ & $0.56 \pm 0.11$ & $0.59 \pm 0.09$ \\
\hline 584 & $0.10 \pm 0.02$ & $0.13 \pm 0.03$ & $0.11 \pm 0.03$ & $0.10 \pm 0.03$ & $0.03 \pm 0.01$ & $0.02 \pm 0.01$ \\
\hline
\end{tabular}

Relative to $\alpha$-tubulin. All data are presented as mean \pm SD.

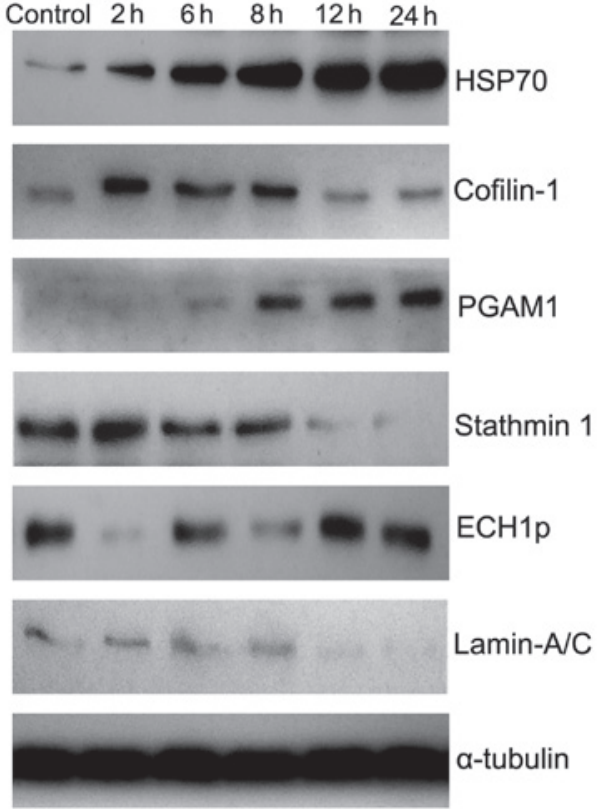

Figure 4. Western blot analysis of differentially expressed proteins following hyperthermia treatment. Heat shock $70 \mathrm{kDa}$ protein (HSP70) was upregulated continuously within $24 \mathrm{~h}$ following hyperthermia treatment, cofilin- 1 was upregulated during the early time period and phosphoglycerate mutase 1 (PGAM1) was upregulated during the middle to late time period. While stathmin 1 was downregulated continuously, $\Delta(3,5)-\Delta(2,4)$-dienoyl-CoA isomerase, mitochondrial (ECH1p) was downregulated during the early time period and lamin $\mathrm{A} / \mathrm{C}$ was downregulated during the middle to late time period.

Among the 44 upregulated proteins, 27 were upregulated continuously, 8 were upregulated during the early time period and 9 were upregulated during the middle to late time period (Table I; Fig. 3Aa-c, a representative figure of the protein expression changes during the three time periods). Among the 13 downregulated proteins, 5 were downregulated continuously, 6 were downregulated during the early time period and two were downregulated during the middle to late time period (Table I; Fig. 3Ad-f).

These protein expression profiles were further confirmed by western blot analysis (Fig. 4). The relative content of these proteins, following heating for $0,2,6,8,12$ and $24 \mathrm{~h}$, are shown in Table III and Fig. 3B $(\mathrm{P}=0.000)$. Therefore, the results demonstrated that protein expression changed in a dynamic manner during hyperthermia-induced Tca8113 cell apoptosis.

\section{Discussion}

The present study investigated protein expression differences in Tca8113 cells following hyperthermia, utilizing fluorescent differential display 2D gel electrophoresis. Changes in expression level were observed in a total of 107 proteins (Fig. 2B), and 57 of these proteins were identified by mass spectrometry analysis. The expression of these proteins varied over different time periods following hyperthermia treatment (Tables I and II) according to the following profiles: 27 were upregulated and 5 were downregulated continuously; 8 were upregulated and 6 were downregulated during the early time period; and 9 were upregulated and 2 were downregulated during the middle to late time period. These results demonstrated that the protein types and expression levels were each upregulated and downregulated during hyperthermia-induced Tca 8113 cell apoptosis. These findings suggest that hyperthermia-induced Tca8113 cell apoptosis is controlled by multiple factors, which include time and regulatory proteins.

Overall, the proteins were grouped into five functional categories. The first functional category consisted of energy metabolism-related proteins. This category included the following proteins: Glyceraldehyde-3-phosphate dehydrogenase (GAPDH); $\alpha$-enolase; inorganic pyrophosphatase; galactokinase; flavin reductase; phosphoglycerate kinase 1; pyridoxine-5'-phosphate oxidase; ATP synthase subunit $\mathrm{d}$, mitochondrial; $26 \mathrm{~S}$ proteasome non-ATPase regulatory subunit 14; protein disulfide-isomerase A3 (PDIA3); glutathione S-transferase P; PGAM1; 3-hydroxyacyl-CoA dehydrogenase type-2 isoform 1; crystal structure of Ufc1, the Ufm1-conjugating enzyme 1, chain A; pyruvate kinase isozymes M1/M2 (PKM1/2); and peroxiredoxin-4, -6 and -2 . The levels of these energy metabolism-related proteins increased following hyperthermic induction. By contrast, the levels of the following energy metabolism-related proteins decreased: ATP synthase subunit $\alpha$, mitochondrial; fructose-bisphosphate aldolase A (ALDOA); mitochondrial succinyl-CoA:3-ketoacid-coenzyme A transferase 1; D-3-phosphoglycerate dehydrogenase; argininosuccinate synthase, mitochondrial; $\Delta(3,5)-\Delta(2,4)$-dienoyl-CoA isomerase, mitochondrial (ECH1p); and hydroxysteroid (17- $\beta$ ) dehydrogenase 10 (HSD17B10). The changes in the expression levels of metabolism-related proteins may lead to impaired tumor cell substance metabolism and energy metabolism, which subsequently affect cell survival. Moreover, it has been 
demonstrated that certain proteins involved in the regulation of cell apoptosis (with the exception of metabolic proteins, such as GAPDH) may be induced to undergo nuclear translocation by ROS, and that increased GAPDH levels are a necessary step for apoptosis (30). For example, PKM2 initiates cell apoptosis by nuclear translocation through a caspase-independent pathway (31). Furthermore, argininosuccinate synthase levels correlate with Bcl-2 levels, and drug-induced cell apoptosis may be enhanced by downregulating argininosuccinate synthase expression (32). In addition, PGAM1 is overexpressed in multiple types of tumors and is involved in tumor formation; it has therefore been suggested that liver cancer cell apoptosis may be induced by the inhibition of PGAM1 (33). Moreover, the increased expression of peroxiredoxin II facilitates tumor cell survival (34), while peroxiredoxin IV inhibits radiation-induced tumor cell apoptosis (35). In the present study, the up- or downregulation of these aforementioned proteases after different time periods following hyperthermic treatment may have been involved in the regulation of apoptosis.

Cytoskeleton-related proteins are another of the five functional categories of proteins. This category includes: Actin, cytoplasmic 2; F-actin-capping protein subunit $\beta$; cofilin-1; tubulin $\beta$ chain; keratin, type I cytoskeletal 10 (CK-10); keratin, type II cytoskeletal 1, 7 and 8 (CK-1,7 and 8); protein 4.1, isoform 3; and far upstream element-binding protein 1 (FBP1). Such proteins were upregulated after different time periods following hyperthermic treatment. The following three cytoskeleton-related proteins were downregulated: Lamin-A/C; vimentin and stathmin 1 (oncoprotein 18). These cytoskeletal proteins are involved in formation of the cell cytoskeleton (involving actin, microtubules, intermediate filaments and the microbeam network) in order to maintain cellular integrity, which is associated with tumorigenesis and the regulation of apoptosis (36-40). Changes in the expression levels of cytoskeleton-related proteins indicate damage to the cytoskeleton, which consequently affects the cell morphology and function. Cytoskeletal proteins have also been demonstrated to be involved in the regulation of apoptosis. Kouzu et al revealed that CK8 is involved in inhibiting several types of drug-induced apoptosis (41). Additionally, inhibiting far upstream element binding protein 1 (FBP1) has been shown to increase hepatocellular carcinoma (HCC) sensitivity towards apoptotic stimulation, which therefore inhibits cell proliferation (42). Furthermore, stathmin has been demonstrated to be downregulated upon the induction of hyperthermia, which also inhibits cell proliferation (43) and results in stathmin phosphorylation and dysfunctional microtubule assembly. Overall, these changes have been demonstrated to induce Jurkat cell apoptosis (44). The present study results indicated that within $24 \mathrm{~h}$ of hyperthermic induction, changes in the expression levels of a variety of cytoskeletal proteins became evident. In particular, changes were observed in the expression levels of stathmin and vimentin, which are proteins associated with tumor growth, while the tumor cell invasion and tumorigenesis dramatically decreased. Therefore, the results indicated that different cytoskeletal proteins are involved in the regulation of apoptosis after different time periods following hyperthermia.

Another of the functional protein categories was that of chaperones. This category included the following proteins: HSP70-1; heat shock cognate $71 \mathrm{kDa}$ protein (HSPA8);
HSP $\beta$-1 (HSPB1/HSP27); PDIA3; T-complex protein 1 subunit $\xi$; and $60 \mathrm{kDa}$ HSP, mitochondrial (HSPD1). Such proteins were upregulated following the induction of hyperthermia. The main function of the chaperones is to maintain cell survival; HSP family members regulate mitochondrial pathway-mediated apoptosis and the death receptor-mediated apoptotic pathway. By blocking the hyperthermia-induced apoptotic pathway, HSPs are able to consequently inhibit apoptosis $(22-25,28)$. In addition, T-complex protein 1 subunit $\zeta$ participates in the assembly of the cytoskeletal proteins actin and tubulin. Moreover, protein disulfide-isomerase A3 is located in the endoplasmic reticulum lumen and acts as a component of the calnexin/calreticulin chaperone complex. Therefore, this protein plays a significant role in calcium homeostasis and regulates free $\mathrm{Ca}^{2+}$, which is able to activate related enzymes and promote apoptosis $(15,16)$. The present study results demonstrated that HSPs are upregulated within $24 \mathrm{~h}$ following hyperthermia, which may result in the regulation of apoptosis through different apoptotic signaling pathways. As PDIA3 and T-complex protein 1 subunit $\zeta$ were also upregulated following hyperthermia, we therefore hypothesized that these two proteins may participate in the regulation of hyperthermia-induced apoptosis.

Transcription factors and protein-synthesis related proteins comprise another of the functional protein categories. This particular category included the following proteins: Scavenger mRNA-decapping enzyme DcpS; crystal structure of Ufc1, the Ufm1-conjugating enzyme 1, chain A; heterogeneous nuclear ribonucleoprotein $\mathrm{H}$; proteasome activator complex subunit 1; deoxyuridine 5'-triphosphate nucleotidohydrolase, mitochondrial (dUTPase); human eukaryotic translation initiation factor $1 \mathrm{~A}$ (eIF1A), chain A; and splicing factor U2AF $65 \mathrm{kDa}$ subunit (U2AF65). These proteins were upregulated after different time periods following hyperthermic treatment. By contrast, eIF5A-1 and the crystal structure of the human eIF5A, chain A were downregulated. It has been confirmed that certain transcription factors and protein synthesis-related proteins are involved in the regulation of apoptosis. For example, the overexpression of heterogeneous nuclear ribonucleoprotein $\mathrm{H}$ (hnRNP H) has been shown to partially counteract apoptosis induced by etoposide, and also to block mammalian STE20-like protein kinase 2 (MST2; proapoptotic MST2 kinase)-mediated apoptosis in cancer cells (45). In addition, eIF5A is able to regulate p53-dependent apoptosis by regulating p53 activity in COS-7 cells (46), while splicing factor U2AF65 subunit participates in Fas-mediated apoptotic regulation (47). Overall, these proteins are up- and downregulated following the induction of hyperthermia, and thus may contribute to hyperthermia-induced apoptotic regulation.

The final functional protein category comprised the cell division- and proliferation-related proteins, including prohibitin, mitotic checkpoint protein BUB3 and BolA-like protein 2 , which were upregulated following hyperthermia treatment. However, one of the proteins in this category, proliferation-associated protein 2G4, was downregulated. Prohibitin is a multifunctional protein that is involved in the following processes: i) the regulation of cell signaling, apoptosis and survival (48); ii) the regulation of cell cycle progression and function as an anti-proliferative protein that is considered to be a tumor suppressor; and iii) the inhibition of cell division 
and tumor growth (49). The present study results indicated that prohibitin is upregulated continuously following hyperthermia treatment. As a tumor suppressor, this protein may participate in the promotion of hyperthermia-induced Tca8113 cell apoptosis.

The present study demonstrated that the expression levels of 57 proteins were dramatically regulated in Tca8113 cells within $24 \mathrm{~h}$ of hyperthermia treatment. The altered proteins were grouped within the following functional classes: energy metabolism-related enzymes, cytoskeleton-related proteins, chaperones, transcription factors and protein synthesis-related proteins and cell division- and proliferation-related proteins. However, no changes were detected in the expression levels of the hyperthermia-induced apoptosis-related proteins that had been previously identified to exhibit such changes, such as p53, Bax, Bak, caspases, cytochrome $C(9,17-20)$, apoptosis-inducing factor (AIF), Bcl-2 and Mcl-1, which may have been due to the low expression of such proteins. Moreover, certain proteins were not detectable by 2D-DIGE. The present study found that the following pro-apoptotic proteins were upregulated: GAPDH, PKM2, eIF5A, prohibitin and mitotic checkpoint protein BUB3. In addition, certain anti-apoptotic proteins were downregulated, including stathmin, vimentin and argininosuccinate synthase. Notably, certain anti-apoptotic proteins were upregulated, including FBP1, hnRNP H, the peroxiredoxins, the HSPs and PGAM1. The expression of 57 proteins was either upregulated or downregulated within $24 \mathrm{~h}$ of hyperthermic treatment. These findings indicate that hyperthermia-induced Tca8113 cell apoptosis is controlled by multiple factors, which include time and regulatory proteins; however, the underlying mechanisms for these changes require further investigation.

\section{Acknowledgements}

The study was supported in part by the National Natural Science Foundation of China (grant no. 81160325), the Ministry of Education Program for New Century Excellent Talents in University (grant no. NCET-07-0388) and the Project of the Department of Science and Technology of Yunnan Province (grant no. 2009CC021).

\section{References}

1. Hildebrandt B and Wust P: The biologic rationale of hyperthermia. Cancer Treat Res 134: 171-184, 2007.

2. van der Zee J: Heating the patient: a promising approach? Ann Oncol 13: 1173-1184, 2002.

3. Yonezawa M, Otsuka T, Matsui N, et al: Hyperthermia induces apoptosis in malignant fibrous histiocytoma cells in vitro. Int J Cancer 66: 347-351,1996.

4. Dewhirst MW, Vujaskovic Z, Jones E and Thrall D: Re-setting the biologic rationale for thermal therapy. Int J Hyperthermia 21: 779-790, 2005.

5. Basile A, Biziato D, Sherbet GV, Comi P and Cajone F: Hyperthermia inhibits cell proliferation and induces apoptosis: relative signaling status of P53, S100A4, and Notch in heat sensitive and resistant cell lines. J Cell Biochem 103: 212-220, 2008.

6. Milleron RS and Bratton SB: 'Heated' debates in apoptosis. Cell Mol Life Sci 64: 2329-2333, 2007.

7. Harmon BV, Takano YS, Winterford CM and Gobé GC: The role of apoptosis in the response of cells and tumours to mild hyperthermia. Int J Radiat Biol 59: 489-501, 1991.

8. Moriyama-Gonda N, Igawa M, Shiina H, Urakami S, Shigeno K and Terashima M: Modulation of heat-induced cell death in PC-3 prostate cancer cells by the antioxidant inhibitor diethyldithiocarbamate. BJU Int 90: 317-325, 2002.
9. Shelton SN, Dillard CD and Robertson JD: Activation of caspase-9, but not caspase-2 or caspase-8, is essential for heat-induced apoptosis in Jurkat cells. J Biol Chem 285: 40525-40533, 2010.

10. Yoo J, Kim HR and Lee YJ: Hyperthermia enhances tumour necrosis factor-related apoptosis-inducing ligand (TRAIL)-induced apoptosis in human cancer cells. Int J Hyperthermia 22: 713-728, 2006.

11. Moulin M, Dumontet C and Arrigo AP: Sensitization of chronic lymphocytic leukemia cells to TRAIL-induced apoptosis by hyperthermia. Cancer Lett 250: 117-127, 2007.

12. Tran S, Meinander A, Holmström T, et al: Heat stress downregulates FLIP and sensitizes cells to Fas receptor-mediated apoptosis. Cell Death Differ 10: 1137-1147, 2003.

13. Zhao QL, Fujiwara Y and Kondo T: Mechanism of cell death induction by nitroxide and hyperthermia. Free Radic Biol Med 40: 1131-1143, 2006.

14. Katschinski DM, Boos K, Schindler SG and Fandrey J: Pivotal role of reactive oxygen species as intracellular mediators of hyperthermia-induced apoptosis. J Biol Chem 275: 21094-21098, 2000.

15. Ahmed K, Zhao QL, Matsuya Y, et al: Enhancement of macrosphelide-induced apoptosis by mild hyperthermia. Int J Hyperthermia 23: 353-361, 2007.

16. Hashimoto T, Shibata M, Ito Y, Nakao KI, Sasaki S and Otsuki Y: Elevated levels of intracellular $\mathrm{Ca}^{2+}$ and apoptosis in human lung cancer cells given heat-shock. Int J Hyperthermia 19: 178-192, 2003.

17. Klostergaard J, Leroux ME, Auzenne E, et al: Hyperthermia engages the intrinsic apoptotic pathway by enhancing upstream caspase activation to overcome apoptotic resistance in MCF-7 breast adenocarcinoma cells. J Cell Biochem 98: 356-369, 2006.

18. Pagliari LJ, Kuwana T, Bonzon C, et al: The multidomain proapoptotic molecules Bax and Bak are directly activated by heat. Proc Natl Acad Sci USA 102: 17975-17980, 2005.

19. Shellman YG, Howe WR, Miller LA, et al: Hyperthermia induces endoplasmic reticulum-mediated apoptosis in melanoma and non-melanoma skin cancer cells. J Invest Dermatol 128: 949-956, 2008.

20. Bonzon C, Bouchier-Hayes L, Pagliari LJ, Green DR and Newmeyer DD: Caspase-2-induced apoptosis requires bid cleavage: a physiological role for bid in heat shock-induced death. Mol Biol Cell 17: 2150-2157, 2006.

21. Beere HM: Death versus survival: functional interaction between the apoptotic and stress-inducible heat shock protein pathways. J Clin Invest 115: 2633-2639, 2005.

22. Banerjee Mustafi S, Chakraborty PK, Dey RS and Raha S: Heat stress upregulates chaperone heat shock protein 70 and antioxidant manganese superoxide dismutase through reactive oxygen species (ROS), p38MAPK, and Akt. Cell Stress Chaperones 14: 579-589, 2009.

23. Shackley DC, Haylett A, Whitehurst C, et al: Comparison of the cellular molecular stress responses after treatments used in bladder cancer. BJU Int 90: 924-932, 2002.

24. Li GC and Calderwood SK: Hyperthermia classic article commentary: 'Re-induction of hsp70 synthesis: an assay for thermotolerance' by Gloria C. Li and Johnson Y. Mak, International Journal of Hyperthermia 1989;5:389-403. Int J Hyperthermia 25: 258-261,2009.

25. Watanabe N, Tsuji N, Akiyama S, et al: Induction of heat shock protein 72 synthesis by endogenous tumor necrosis factor via enhancement of the heat shock element-binding activity of heat shock factor 1. Eur J Immunol 27: 2830-2834, 1997.

26. Strasser A and Anderson RL: Bcl-2 and thermotolerance cooperate in cell survival. Cell Growth Differ 6: 799-805, 1995.

27. Stankiewicz AR, Livingstone AM, Mohseni N and Mosser DD: Regulation of heat-induced apoptosis by Mcl-1 degradation and its inhibition by Hsp70. Cell Death Differ 16: 638-647, 2009.

28. Jiang W, Bian L, Ma LJ, Tang RZ, Xun S and He YW: Hyperthermia-induced apoptosis in Tca8113 cells is inhibited by heat shock protein 27 through blocking phospholipid scramblase 3 phosphorylation. Int J Hyperthermia 26: 523-537, 2010.

29. Tannu NS and Hemby SE: Two-dimensional fluorescence difference gel electrophoresis for comparative proteomics profiling. Nat Protoc 1: 1732-1742, 2006.

30. Hara MR, Agrawal N, Kim SF, et al: S-nitrosylated GAPDH initiates apoptotic cell death by nuclear translocation following Siah1 binding. Nat Cell Biol 7: 665-674, 2005. 
31. Steták A, Veress R, Ovádi J, Csermely P, Kéri G and Ullrich A: Nuclear translocation of the tumor marker pyruvate kinase M2 induces programmed cell death. Cancer Res 67: 1602-1608, 2007.

32. Goodwin BL, Solomonson LP and Eichler DC: Argininosuccinate synthase expression is required to maintain nitric oxide production and cell viability in aortic endothelial cells. J Biol Chem 279: 18353-18360, 2004.

33. Ren F, Wu H, Lei Y, et al: Quantitative proteomics identification of phosphoglycerate mutase 1 as a novel therapeutic target in hepatocellular carcinoma. Mol Cancer 9: 81, 2010.

34. Lee KW, Lee DJ, Lee JY, Kang DH, Kwon J and Kang SW: Peroxiredoxin II restrains DNA damage-induced death in cancer cells by positively regulating JNK-dependent DNA repair. J Bio Chem 286: 8394-8404, 2011.

35. Park JJ, Chang HW, Jeong EJ, et al: Peroxiredoxin IV protects cells from radiation-induced apoptosis in head-and-neck squamous cell carcinoma. Int J Radiat Oncol Biol Phys 73: 1196-1202, 2009.

36. Lundkvist A, Reichenbach A, Betsholtz C, Carmeliet P Wolburg $\mathrm{H}$ and Pekny M: Under stress, the absence of intermediate filaments from Müller cells in the retina has structural and functional consequences. J Cell Sci 117: 3481-3488, 2004.

37. Lau AT and Chiu JF: The possible role of cytokeratin 8 in cadmium-induced adaptation and carcinogenesis. Cancer Res 67: 2107-2113, 2007

38. Bhat KM and Setaluri V: Microtubule-associated proteins as targets in cancer chemotherapy. Clin Cancer Res 13: 2849-2854, 2007.

39. Prasad S, Soldatenkov Va, Srinivasarao G and Dritschilo A Intermediate filament proteins during carcinogenesis and apoptosis (Review). Int J Oncol 14: 563-570, 1999.

40. Rana S, Maples PB, Senzer N and Nemunaitis J: Stathmin 1: a novel therapeutic target for anticancer activity. Expert Rev Anticancer Ther 8: 1461-1470, 2008.
41. Kouzu Y, Uzawa K, Koike H, et al: Overexpression of stathmin in oral squamous-cell carcinoma: correlation with tumour progression and poor prognosis. Br J Cancer 94: 717-723, 2006.

42. Nakamura K, Zhang X, Kuramitsu Y, et al: Analysis on heat stress-induced hyperphosphorylation of stathmin at serine 37 in Jurkat cells by means of two-dimensional gel electrophoresis and tandem mass spectrometry. J Chromatogr A 1106: 181-189, 2006.

43. Cajone F and Sherbet GV: Stathmin is involved in S100A4-mediated regulation of cell cycle progression. Clin Exp Metastasis 17: 865-871, 1999.

44. Rabenhorst U, Beinoraviciute-Kellner R, Brezniceanu ML, et al: Overexpression of the far upstream element binding protein 1 in hepatocellular carcinoma is required for tumor growth. Hepatology 50: 1121-1129, 2009.

45. Rauch J, O'Neill E, Mack B, et al: Heterogeneous nuclear ribonucleoprotein H blocks MST2-mediated apoptosis in cancer cells by regulating A-Raf transcription. Cancer Res 70: 1679-1688, 2010.

46. Li AL, Li HY, Jin BF, et al: A novel eIF5A complex functions as a regulator of p53 and p53-dependent apoptosis. J Biol Chem 279: 49251-49258, 2004.

47. Izquierdo JM: Fas splicing regulation during early apoptosis is linked to caspase-mediated cleavage of U2AF65. Mol Biol Cell 19: 3299-3307, 2008.

48. Zhu B, Zhai J, Zhu H and Kyprianou N: Prohibitin regulates TGF-beta induced apoptosis as a downstream effector of Smad-dependent and -independent signaling. Prostate 70: 17-26, 2010.

49. Wang S, Nath N, Adlam M and Chellappan S: Prohibitin, a potential tumor suppressor, interacts with RB and regulates E2F function. Oncogene 18: 3501-3510, 1999. 